\title{
An exact solution of spherical mean-field plus a special separable pairing model
}

\author{
Lianrong Dai, ${ }^{1}$ Feng Pan*,1,2 and J. P. Draayer ${ }^{2}$ \\ ${ }^{1}$ Department of Physics, Liaoning Normal University, Dalian 116029, China \\ ${ }^{2}$ Department of Physics and Astronomy, Louisiana State University, Baton Rouge, LA 70803-4001, USA
}

\begin{abstract}
An exact solution of nuclear spherical mean-field plus a special orbit-dependent separable pairing model is studied, of which the separable pairing interaction parameters are obtained by a linear fitting in terms of the single-particle energies considered. The advantage of the model is that, similar to the standard pairing case, it can be solved easily by using the extended Heine-Stieltjes polynomial approach. With the analysis of the model in the $d s$ - and $p f$-shell subspace, it is shown that this special separable pairing model indeed provides similar pair structures of the model with the original separable pairing interaction, and is obviously better than the standard pairing model in many aspects.
\end{abstract}

PACS numbers: 21.60.Fw, 03.65.Fd, 02.20.Qs, 02.30.Ik

\section{INTRODUCTION}

Pairing interaction has been considered of importance in many branches of physics [1-4]. In nuclear physics, the pairing interaction is key to elucidate ground state and low-energy spectroscopic properties of nuclei, such as binding energies, oddeven effects, single-particle occupancies, excitation spectra, and moments of inertia, etc [5-7]. Though the Bardeen-CooperSchrieffer (BCS) [1] and the more refined Hartree-Fock-Bogolyubov (HFB) approximations provide simple and clear pictures in nuclei $[6,8,9]$, tremendous efforts have been made in finding accurate solutions to the problem [10-15] to overcome serious drawbacks in the BCS and the HFB resulting from particle number-nonconservation effects [13, 15-17]. It is known that either spherical or deformed mean-field plus the standard orbit independent pairing interaction can be solved exactly by using the Gaudin-Richardson method [18-20]. It has been shown that the set of Gaudin-Richardson equations can be solved easily by using the extended Heine-Stieltjes polynomial approach [21-24]. The deformed and spherical mean-field plus the extended pairing models have also been proposed, which can be solved more easily than the standard pairing model, especially when both the number of valence nucleon pairs and the number of single-particle orbits are large [25, 26].

The separable pairing problem was studied in [27], in which the single-particle energies are all degenerate. A case with two non-degenerate levels was analyzed in [28]. General non-degenerate cases were also considered previously [29, 30]. However, the expressions are complicated with the specially chosen constraints on the single-particle energies and the separable pairing interaction parameters, which are difficult to be solved numerically. In this work, we show that there are special cases of [29, 30], of which the solutions can be obtained easily by using the algebraic Bethe ansatz. It should be pointed out that solutions of the special separable pairing cases considered in this work were also derived previously by using the generalized Gaudin algebraic formalism [31, 32]. These special cases belong to a special family of the hyperbolic Richardson-Gaudin models studied in $[31,32]$.

\section{THE MODEL AND ITS EXACT SOLUTION}

The Hamiltonian of a mean-field plus the separable pairing model may be written as

$$
\hat{H}=\sum_{t=1}^{p} \epsilon_{j_{t}} \hat{N}_{j_{t}}+\hat{H}_{\mathrm{P}}=\sum_{t=1}^{p} \epsilon_{j_{t}} \hat{N}_{j_{t}}-G \sum_{1 \leq t, t^{\prime} \leq p} c_{j_{t}} c_{j_{t}} S_{j_{t}}^{+} S_{j_{t^{\prime}}}^{-},
$$

* The corresponding author's e-mail: daipan@dlut.edu.cn 
where $p$ is the total number of orbits considered, $\left\{\epsilon_{j_{t}}\right\}(t=1,2, \cdots, p)$ is a set of single-particle energies generated from any mean-field theory, such as those of the shell model, $\hat{N}_{j}=\sum_{m} a_{j m}^{\dagger} a_{j m}$ and $S_{j}^{+}=\sum_{m>0}(-1)^{j-m} a_{j m}^{\dagger} a_{j-m}^{\dagger}$, in which $a_{j m}^{\dagger}\left(a_{j m}\right)$ is the creation (annihilation) operator for a nucleon with angular momentum quantum number $j$ and that of its projection $m, G$ and $\left\{c_{j_{t}}\right\}(t=1,2, \cdots, p)$ are the separable pairing interaction parameters, which are all assumed to be real in the following.

The set of local operators $\left\{S_{j_{t}}^{-}, S_{j_{t}}^{+}, \hat{N}_{j_{t}}\right\}(t=1,2, \cdots, p)$, where $S_{j_{t}}^{-}=\left(S_{j_{t}}^{+}\right)^{\dagger}$, generate $p$ copies of SU(2) algebra satisfying the commutation relations

$$
\left[\hat{N}_{j_{t}} / 2, S_{j_{t^{\prime}}}^{-}\right]=-\delta_{t t^{\prime}} S_{j_{t}}^{-},\left[\hat{N}_{j_{t}} / 2, S_{j_{t^{\prime}}}^{+}\right]=\delta_{t t^{\prime}} S_{j_{t}}^{+},\left[S_{j_{t}}^{+}, S_{j_{t^{\prime}}}^{-}\right]=2 \delta_{t t^{\prime}} S_{j_{t}}^{0},
$$

where $S_{j_{t}}^{0}=\left(\hat{N}_{j_{t}}-\Omega_{j_{t}}\right) / 2$ with $\Omega_{j_{t}}=j_{t}+1 / 2$.

Let

$$
S^{+}(x)=\sum_{t=1}^{p} \frac{1}{2 \epsilon_{j_{t}}-x} c_{j_{t}} S_{j_{t}}^{+}
$$

where $x$ is the spectral parameter to be determined, which was used in [29, 30]. According to the commutation relations given in (2), we have

$$
\begin{gathered}
{\left[\sum_{t} \epsilon_{j_{t}} \hat{N}_{j_{t}}, S^{+}(x)\right]=\sum_{t} \frac{2 \epsilon_{j_{t}}}{2 \epsilon_{j_{t}}-x} c_{j_{t}} S_{j_{t}}^{+}=\sum_{t} c_{j_{t}} S_{j_{t}}^{+}+x S^{+}(x),} \\
{\left[\hat{H}_{\mathrm{P}}, S^{+}(x)\right]=G \sum_{t^{\prime}} c_{j_{t^{\prime}}} S_{j_{t^{\prime}}}^{+} \sum_{t} \frac{2 S_{j_{t}}^{0}\left(c_{j_{t}}\right)^{2}}{2 \epsilon_{j_{t}}-x}=G \sum_{j_{t^{\prime}}} c_{j_{t^{\prime}}} S_{j_{t^{\prime}}}^{+} \Lambda_{0}(x),}
\end{gathered}
$$

where $\Lambda_{0}(x)=\sum_{t} \frac{2 S_{j_{t}}^{0}\left(c_{j_{t}}\right)^{2}}{2 \epsilon_{j_{t}}-x}$

$$
\left[\left[\hat{H}_{\mathrm{P}}, S^{+}(x)\right], S^{+}(y)\right]=2 G \sum_{t^{\prime}} c_{j_{t^{\prime}}} S_{j_{t^{\prime}}}^{+} \sum_{t} \frac{\left(c_{j_{t}}\right)^{2}}{\left(2 \epsilon_{j_{t}}-x\right)\left(2 \epsilon_{j_{t}}-y\right)} c_{j_{t}} S_{j_{t}}^{+} .
$$

It is easy to check that the Hamiltonian (1) can be solved exactly by setting

$$
\left(c_{j_{t}}\right)^{2}=g_{1} \epsilon_{j_{t}}+g_{2} \forall t
$$

where $g_{1}$ and $g_{2}$ are parameters to be determined. It is obvious that the model becomes the standard pairing case when $g_{2}$ is a constant and $g_{1}=0$. It should be stated that the solvability of (1) with the constraints (7) has been verified in [31, 32] by using the generalized Gaudin algebraic technique.

By using (7), Eq. (6) can be rewritten as

$$
\begin{gathered}
S^{+}(x, y)=\left[\left[\hat{H}_{\mathrm{P}}, S^{+}(x)\right], S^{+}(y)\right]=2 G \sum_{t^{\prime}} c_{j_{t^{\prime}}} S_{j_{t^{\prime}}}^{+} \sum_{t} \frac{\left(g_{1} \epsilon_{j_{t}}+g_{2}\right) c_{j_{t}}}{\left(2 \epsilon_{j_{t}}-x\right)\left(2 \epsilon_{j_{t}}-y\right)} S_{j_{t}}^{+}= \\
G \sum_{t^{\prime}} c_{j_{t^{\prime}}} S_{j_{t^{\prime}}}^{+}\left(\frac{g_{1}}{x-y}\left(x S^{+}(x)-y S^{+}(y)\right)+\frac{2 g_{2}}{x-y}\left(S^{+}(x)-S^{+}(y)\right)\right),
\end{gathered}
$$

which, together with Eq. (4), is the key to solve the problem.

Let $|0\rangle$ be the vacuum state satisfying $a_{j_{t} m}|0\rangle=0 \forall t$. For the seniority $v_{j_{t}} \neq 0$ case, the pairing vacuum states are denoted as $\left|v_{j_{t}} \eta_{j_{t}} J_{t} M_{t}\right\rangle$ satisfying $S_{j_{t}}^{-}\left|v_{j_{t}} \eta_{j_{t}} J_{t} M_{t}\right\rangle=0$, where $J_{j_{t}}$ and $M_{J_{j_{t}}}$ are the angular momentum quantum number and that of its third component, respectively, and $\eta_{j_{t}}$ is the multiplicity label needed to distinguish different possible ways of $v_{j_{t}}$ particles coupled to the angular momentum $J_{j_{t}}$ under the anti-symmetric irreducible representation $\left\langle 1^{v_{j_{t}}}\right\rangle$ of $\operatorname{Sp}\left(2 j_{t}+1\right) \downarrow \mathrm{O}_{t}(3)$. Thus, a 
pairing vacuum state of a $p$-orbit system with the total number of particles $N=\sum_{t} v_{j_{t}}$, and the total angular momentum $J$ can be expressed as $|J M\rangle \equiv\left|v_{j_{1}} \eta_{j_{1}}, \cdots, v_{j_{p}} \eta_{j_{p}} ; \tau\left(J_{j_{1}} \times J_{j_{2}} \times \cdots \times J_{j_{p}}\right) J M\right\rangle$, in which the angular momentum coupling with $J_{j_{1}} \otimes \cdots \otimes J_{j_{p}} \downarrow J$ should be made, and $\tau$ is the multiplicity label needed to resolve the multiplicity of $J$ in the coupling.

Thus, similar to the standard pairing model, $k$-pair eigenstate of (1) may be expressed as

$$
|\zeta, k ; J M\rangle=S^{+}\left(x_{1}^{(\zeta)}\right) \cdots S^{+}\left(x_{k}^{(\zeta)}\right)|J M\rangle
$$

Using Eqs. (4) -(6) and (8), we can directly check that

$$
\begin{gathered}
\sum_{j} \epsilon_{j} \hat{N}_{j}|\zeta, k ; J M\rangle= \\
\left(\sum_{t} c_{j_{t}} S_{j_{t}}^{+}+x_{1}^{(\zeta)} S^{+}\left(x_{1}^{(\zeta)}\right)\right) S^{+}\left(x_{2}^{(\zeta)}\right) \cdots S^{+}\left(x_{k}^{(\zeta)}\right)|J M\rangle+ \\
\cdots+S^{+}\left(x_{1}^{(\zeta)}\right) \cdots S^{+}\left(x_{k-1}^{(\zeta)}\right)\left(\left(\sum_{t} c_{j_{t}} S_{j_{t}}^{+}+x_{k}^{(\zeta)} S^{+}\left(x_{k}^{(\zeta)}\right)\right)|J M\rangle\right.
\end{gathered}
$$

and

$$
\begin{gathered}
\hat{H}_{\mathrm{P}}|\zeta, k ; J M\rangle=G \sum_{t^{\prime}} c_{j_{t^{\prime}}} S_{j_{t^{\prime}}}^{+}\left(\Lambda_{0}\left(x_{1}^{(\zeta)}\right) S^{+}\left(x_{2}^{(\zeta)}\right) \cdots S^{+}\left(x_{k}^{(\zeta)}\right)+\right. \\
\left.\cdots+S^{+}\left(x_{1}^{(\zeta)}\right) \cdots S^{+}\left(x_{k-1}^{(\zeta)}\right) \Lambda_{0}\left(x_{k}^{(\zeta)}\right)\right)|J M\rangle \\
+\sum_{t^{\prime}} c_{j_{t^{\prime}}} S_{j_{t^{\prime}}}^{+}\left(S^{+}\left(x_{1}^{(\zeta)}, x_{2}^{(\zeta)}\right) S^{+}\left(x_{3}^{(\zeta)}\right) \cdots S^{+}\left(x_{k}^{(\zeta)}\right)+S^{+}\left(x_{1}^{(\zeta)}, x_{3}^{(\zeta)}\right) S^{+}\left(x_{2}^{(\zeta)}\right) S^{+}\left(x_{4}^{(\zeta)}\right) \cdots S^{+}\left(x_{k}^{(\zeta)}\right)\right. \\
+\cdots+S^{+}\left(x_{1}^{(\zeta)}, x_{k}^{(\zeta)}\right) S^{+}\left(x_{2}^{(\zeta)}\right) \cdots S^{+}\left(x_{k-1}^{(\zeta)}\right)+ \\
\cdots+S^{+}\left(x_{k}^{(\zeta)}, x_{1}^{(\zeta)}\right) S^{+}\left(x_{2}^{(\zeta)}\right) \cdots S^{+}\left(x_{k-1}^{(\zeta)}\right)+S^{+}\left(x_{k}^{(\zeta)}, x_{2}^{(\zeta)}\right) S^{+}\left(x_{3}^{(\zeta)}\right) \cdots S^{+}\left(x_{k-1}^{(\zeta)}\right)+ \\
\left.\cdots+S^{+}\left(x_{k}^{(\zeta)}, x_{k-1}^{(\zeta)}\right) S^{+}\left(x_{2}^{(\zeta)}\right) \cdots S^{+}\left(x_{k-2}^{(\zeta)}\right)\right)|J M\rangle .
\end{gathered}
$$

By using Eqs. (10) -(11), one can prove that the eigen-equation $\hat{H}|\zeta, k ; J M\rangle=E_{k}^{(\zeta)}|\zeta, k ; J M\rangle$ is fulfilled when and only when

$$
\sum_{t=1}^{p} \frac{\left(g_{1} \epsilon_{j_{t}}+g_{2}\right)\left(\Omega_{j_{t}}-v_{j_{t}}\right)}{2 \epsilon_{j_{t}}-x_{i}^{(\zeta)}}+\sum_{l \neq i} \frac{g_{1} x_{l}^{(\zeta)}+2 g_{2}}{x_{i}^{(\zeta)}-x_{l}^{(\zeta)}}=1 / G \text { for } i=1,2, \cdots k
$$

with the corresponding eigen-energy

$$
E_{k}^{(\zeta)}=\sum_{t=1}^{p} \epsilon_{j_{t}} v_{j_{t}}+\sum_{i=1}^{k} x_{i}^{(\zeta)}
$$

in which $\sum_{t=1}^{p} \epsilon_{j_{t}} v_{j_{t}}$ is contributed from particles in the pairing vacuum. In the following, we assume that $g_{1}$ and $g_{2}$ are real and spectral parameter independent constants, which includes the two-orbit solvable model [28] as a special case.

According to Heine-Stieltjes correspondence [21, 22], for nonzero $G$, zeros $\left\{x_{i}^{(\zeta)}\right\}$ of the extended Heine-Stieltjes polynomials $y_{k}(x)$ of degree $k$ are roots of Eq. (12), where $y_{k}(x)$ should satisfy the following second-order Fuchsian equation:

$$
A(x) y_{k}^{\prime \prime}(x)+B(x, k) y_{k}^{\prime}(x)-V(x, k) y_{k}(x)=0 \text {. }
$$

Here, $A(x)=\frac{1}{2}\left(g_{1} x+2 g_{2}\right) \prod_{t=1}^{p}\left(2 \epsilon_{j_{t}}-x\right)$ is a polynomial of degree $p+1$, the polynomial $B(x, k)$ of degree $p-1$ is given as

$$
B(x, k) / A(x)=\frac{2}{g_{1} x+2 g_{2}} \sum_{t=1}^{p} \frac{\left(g_{1} \epsilon_{j_{t}}+g_{2}\right)\left(\Omega_{j_{t}}-v_{j_{t}}\right)}{2 \epsilon_{j_{t}}-x}-\frac{2(k-1) G g_{1}+2}{\left(g_{1} x+2 g_{2}\right) G},
$$

where the identities 


$$
\sum_{1 \leq l(\neq i) \leq k} \frac{x_{l}^{(\zeta)}}{x_{i}^{(\zeta)}-x_{l}^{(\zeta)}}=\sum_{1 \leq l(\neq i) \leq k} \frac{\left(x_{l}^{(\zeta)}-x_{i}^{(\zeta)}\right)+x_{i}^{(\zeta)}}{x_{i}^{(\zeta)}-x_{l}^{(\zeta)}}=x_{i}^{(\zeta)} \sum_{1 \leq l(\neq i) \leq k} \frac{1}{x_{i}^{(\zeta)}-x_{l}^{(\zeta)}}-(k-1)
$$

and

$$
\frac{y_{k}^{\prime \prime}\left(x_{i}\right)}{y_{k}^{\prime}\left(x_{i}\right)}=\sum_{1 \leq l(\neq i) \leq k} \frac{2}{x_{i}-x_{l}}
$$

are used in obtaining (15) from (12), and $V(x)$ is a Van Vleck polynomial of degree $p-1$, which is determined according to Eq. (14). Therefore, the polynomial approach for the standard pairing model proposed in [22, 23] applies to the separable pairing model as well. Since the model with arbitrary separable pairing interaction parameters $\left\{c_{j}\right\}$ can always be diagonalized numerically, it has been verified by using a modified code of [23] according to (14) and (15) that the Bethe ansatz solutions (9), (12), and (13) coincide with those obtained from the progressive diagonalization scheme [33], which justifies the exact solvability of the model by using the Bethe ansatz method and the generalized Richardson-Gaudin algebraic technique shown in $[31,32]$.

\section{APPLICATION TO THE $d s$ AND $p f$ SHELL}

In the previous work [34], the deformed mean-field plus the special separable pairing model with $c_{i}^{2}=\alpha-\epsilon_{i}$, where $\epsilon_{i}$ are single-particle energies generated from the deformed mean-field, and $\alpha$ is a constant related to an interaction cutoff, was used to describe well deformed nuclei. It is shown in [34] that their model results excellently reproduce the Gogny self-consistent mean-field calculations in the canonical basis. To demonstrate the solution of the present model within a truncated spherical shell model subspace, we consider two examples restricted within the seniority-zero subspace. One is the $d s$-shell with 3 orbitals $0 d_{5 / 2}, 1 s_{1 / 2}$, and $0 d_{3 / 2}$, of which the single-particle energies are provided in [35]. The pairing interaction matrix elements in the $\left\{S_{j}^{+}\right\}$basis with $\hat{H}_{\text {Pairing }}=\sum_{j j^{\prime}} G_{2 j, 2 j^{\prime}} S_{j}^{+} S_{j^{\prime}}^{-}$for this case are obtained from the $J=0$ and $T=1$ two-body matrix elements of the SDPF-NR interaction shown in [35]. Another is the $p f$-shell with 4 orbitals $1 p_{3 / 2}, 1 p_{1 / 2}, 0 f_{7 / 2}$, and $0 f_{5 / 2}$. The pairing interaction matrix elements in the $\left\{S_{j}^{+}\right\}$basis in the $p f$-shell are obtained from the $J=0$ and $T=1$ two-body matrix elements of GXPF1 interaction [36], in which the single-particle energies are also provided. The mass scaling factors, $(A / 18)^{1 / 3}$ for the $d s$-shell and $(A / 42)^{-0.3}$ for the $p f$-shell, were not included in our analysis. Table I provides the single-particle energies and the pairing interaction matrix elements in the $\left\{S_{j}^{+}\right\}$basis for these two cases. According to the method used in [27], the separable pairing interaction parameters may be determined from the two-particle ground state of the mean-field plus the $\hat{H}_{\text {Pairing }}$, of which the expansion coefficients of the two-particle ground state in terms of $\left\{S_{j}^{+}\right\}$may be taken to be the corresponding separable pairing interaction parameter $c_{j}$ of the spherical mean-field plus the separable pairing model (SP). Then, we use a linear fit of $\left\{c_{j}^{2}\right\}$ by the single-particle energies with $1 \geq \tilde{c}_{j}^{2}=g_{1} \epsilon_{j}+g_{2} \geq 0$ according to the constraint shown in (7), with which the model will be called the special separable pairing model (SSP). The best linear fit yields $\tilde{c}_{j}^{2}=0.1524-0.067 \epsilon_{j}$ for the $d s$-shell and $\tilde{c}_{j}^{2}=0.139-0.022 \epsilon_{j}$ for the $p f$-shell, where the parameter $g_{1}$ is in $(\mathrm{MeV})^{-1}$. The SP and the SSP parameters thus determined are all provided in Table I. The overall strength $G$ in the SP was adjusted to reproduce the same two-particle ground state energy, which is thus fixed though it also varies with $k$. Since the overall pairing strength varies with the number of pairs $k$, the overall pairing interaction strength in the SSP, which is denoted as $\tilde{G}$, was then adjusted to reproduce the same ground state energy of the SP for a given $k$. The pairing excitation spectrum generated by the SSP in each case is compared with that generated by the SP for a given number of pairs $k$ up to the half-filling. The general SP Hamiltonian (1) was numerically diagonalized by using the progressive diagonalization scheme [33] based on the solution of the pure separable pairing part [27] in the $d s$ - or $p f$-shell subspace for a given number of pairs $k$, which is practically easier than the procedure shown in [29, 30]. The results of the orbit-independent standard pairing model (SPM) with $\left\{c_{j}=1\right\}$ and the overall pairing interaction strength $G_{s}$, of which the exact solution is well known [19, 20, 22-24], are also shown for comparison. We observe that both the value of $\tilde{G}$ in the SSP and that of $G_{s}$ in the standard pairing model (SPM) decreases with the increasing of the number of pairs $k$ quadratically. Moreover, as shown in Fig. 1, larger value of $G_{s}$ is needed in the $d s$-shell than that in the $p f$-shell, while $\tilde{G}$ in the SSP for both shells behaves quite the same. 
TABLE I: The single-particle energies $\epsilon_{j}$ (in $\mathrm{MeV}$ ) and the pairing interaction matrix elements $G_{2 j, 2 j^{\prime}}$ (in $\mathrm{MeV}$ ) in the $\left\{S_{j}^{+}\right\}$basis for the $d s$ and $p f$-shell deduced from $[35,36]$, the SP parameters $\left\{c_{j}\right\}$ and $G$ (in $\mathrm{MeV}$ ), and the SSP parameters $\left\{\tilde{c}_{j}\right\}$.

\begin{tabular}{llllll}
\hline \hline The $d s$-shell & $\epsilon_{5 / 2}=-3.70$ & $\epsilon_{1 / 2}=-2.92$ & $\epsilon_{3 / 2}=1.90$ & & \\
& $G_{55}=-0.915$ & $G_{11}=-1.075$ & $G_{33}=-0.410$ & & \\
& $G_{51}=0.1212$ & $G_{53}=-0.355$ & $G_{31}=0.00$ & & \\
& $c_{5 / 2}=0.99583$ & $c_{1 / 2}=-0.06334$ & $c_{3 / 2}=0.06562$ & $G=0.945$ & \\
& $\tilde{c}_{5 / 2}=0.63269$ & $\tilde{c}_{1 / 2}=-0.58995$ & $\tilde{c}_{3 / 2}=0.15843$ & & \\
\hline The $p f$-shell & $\epsilon_{7 / 2}=-8.624$ & $\epsilon_{3 / 2}=-5.6793$ & $\epsilon_{1 / 2}=-4.137$ & $\epsilon_{5 / 2}=-1.3829$ & \\
& $G_{77}=-1.21295$ & $G_{73}=-0.50728$ & $G_{75}=-0.79859$ & $G_{71}=-0.3800$ & $G_{33}=-1.1165$ \\
& $G_{35}=-1.01711$ & $G_{31}=-2.11114$ & $G_{55}=-0.8054$ & $G_{51}=-0.934499$ & $G_{11}=-0.89380$ \\
& $c_{7 / 2}=0.94638$ & $c_{3 / 2}=0.23101$ & $c_{1 / 2}=0.123105$ & $c_{5 / 2}=0.18933$ & $G=1.590$ \\
& $\tilde{c}_{7 / 2}=0.57335$ & $\tilde{c}_{3 / 2}=0.513755$ & $\tilde{c}_{1 / 2}=0.47960$ & $\tilde{c}_{5 / 2}=0.41161$ & \\
\hline \hline
\end{tabular}

Table II shows all pairing excitation energies of the model in the $d s$ - and $p f$-shell up to the half-filling. The quality of the results in comparison to those obtained from the SP is measured by

$$
\chi=\sqrt{\frac{1}{D-1} \sum_{\zeta=1}^{D}\left(E_{k}^{(\zeta)}(\mathrm{SP})-E_{k}^{(\zeta)}\right)^{2}},
$$

where $D$ is the dimension of the entire $k$-pair subspace in the $d s$ - or $p f$-shell considered, $E_{k}^{(\zeta)}$ is the $\zeta$-th pairing excitation energy produced by either the SSP or the SPM. As shown in Table II and Fig. 2, for any $k$, the SSP is better than the SPM. However, the deviation of both models from the SP becomes larger with the increasing of the number of pairs. A further measure of the model quality is also illustrated in Fig. 2, in which the ground-state overlap-square of the SSP or the SPM with the corresponding one of the SP defined by $\rho=\left|\langle\zeta=1, k \mid \zeta=1, k\rangle_{\mathrm{SP}}\right|^{2}$, where $|\zeta=1 ; k\rangle_{\mathrm{SP}}$ is the ground state of the SP, and $|\zeta=1 ; k\rangle$ is either the ground state of the SSP or that of SPM, is also shown. It is obvious that the $\chi$ value of both the SSP and the SMP increases with the increasing of $k$. With the increasing of the total number of orbits considered, the overlap increases. As shown in Fig. 2, the value of $\rho$ is always greater than $80 \%$ up to the half-filling in the $p f$-shell, which involves 4 orbits. In all cases, the $\chi$ value of the SSP is noticeably smaller than that of the SPM, and the overlap-square of the SSP is always a little greater than the corresponding one of the SPM. All the results indicate that the SSP is a better approximation to the original separable pairing model [27] than the standard pairing model (SPM), though both the SSP and SPM produce the SP spectra globally well, especially for the low-lying part of the spectra.
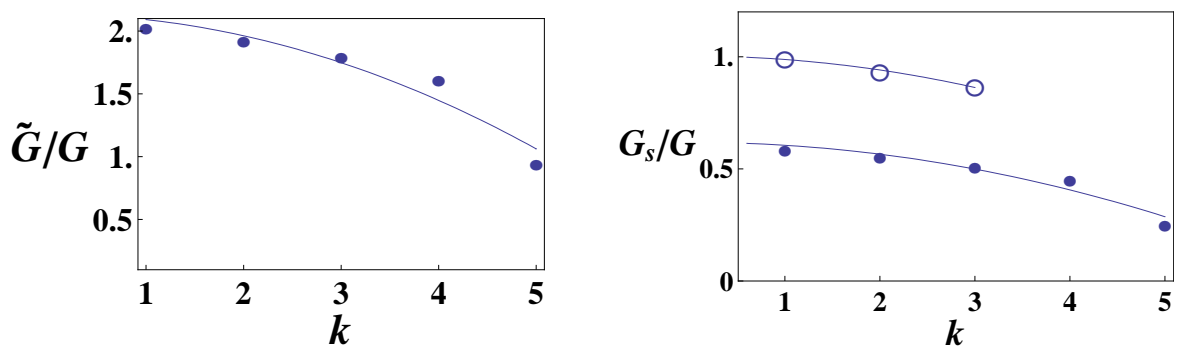

FIG. 1: The overall pairing strength $\tilde{G}$ of the SSP and $G_{s}$ of the SPM as functions of $k$, where the parameter $G$ in the SP is fixed according to the two-particle ground state of the shell model mean-field plus the effective $J=0$ and $T=1$ two-body interaction [35, 36], where $\tilde{G}$ denoted by the solid dot for each $k$ in both the $d s$ - and the $p f$-shell shown in the left panel, the open circle denotes the $G_{s}$ value for each $k$ in the $d s$-shell, the solid dot in the right panel denotes that in the $p f$-shell, and the curve in each case is obtained by a $k^{2}$-fitting to guide to the eye. 

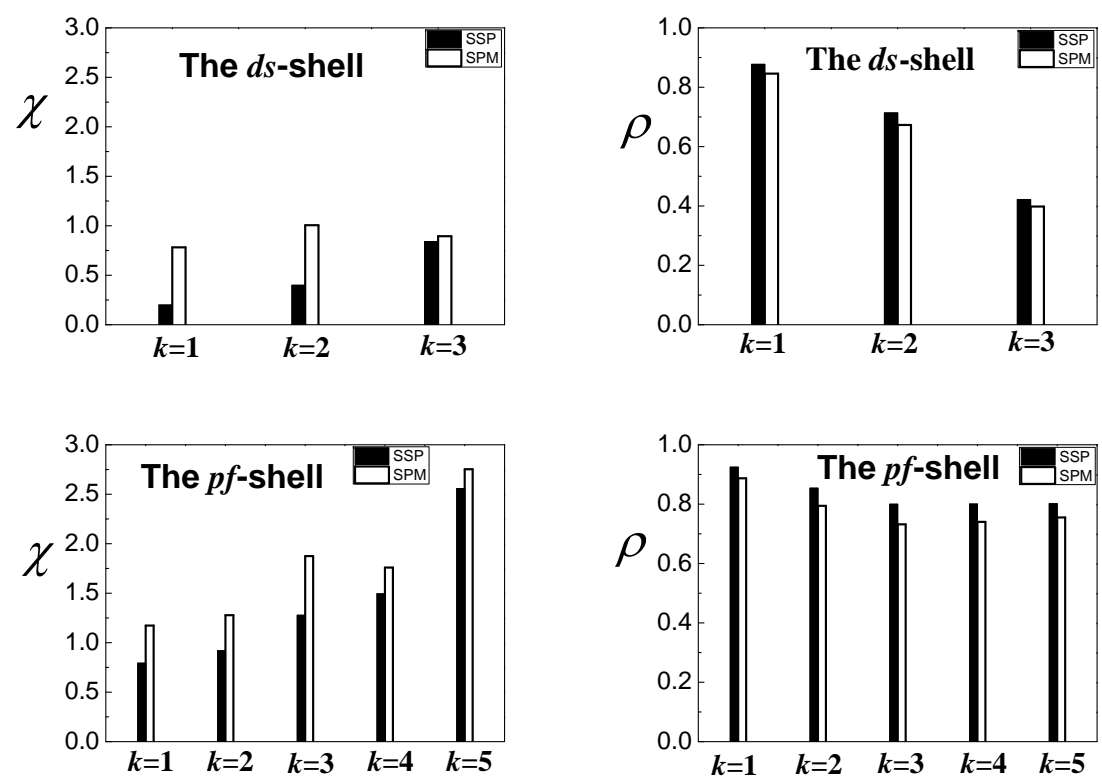

FIG. 2: The root-mean-square deviation in excitation energies $\chi$ (in MeV) defined in (18) and the ground state overlap-square with that of the SP for a given number of pairs $k$ in the $d s$ - and $p f$-shell, where the solid bar represents the result of the SSP, and the hollow bar represents that of the SPM. 
TABLE II: Comparison of pairing excitation energies $E_{k}^{(\zeta)}$ (in $\mathrm{MeV}$ ) of the seniority-zero case obtained from the SSP and the SPM with those of the SP for a given number of pairs $k$ up to the half-filling, where the overall strength $\tilde{G}$ in the SSP or $G_{s}$ in the SPM is adjusted to produce the same ground state energy $E_{k}^{(\zeta=1)}$ for any $k$.

\begin{tabular}{crrrrrrrrr}
\hline \hline & \multicolumn{3}{c}{$k=1$} & & & $k=2$ & \multicolumn{3}{c}{$k=3$} \\
\hline The $d s$-shell & SP & SSP & SPM & SP & SSP & SPM & SP & SSP & SPM \\
\hline$\zeta=1$ & -10.22 & -10.22 & -10.22 & -18.56 & -18.56 & -18.56 & -25.03 & -25.03 & -25.03 \\
$\zeta=2$ & -5.84 & -6.09 & -6.12 & -16.05 & -15.33 & -15.27 & -24.38 & -22.91 & -22.83 \\
$\zeta=3$ & 3.79 & 3.72 & 2.72 & -6.42 & -6.30 & -7.03 & -14.76 & -14.28 & -14.91 \\
$\zeta=4$ & & & & -2.05 & -2.36 & -3.45 & -12.26 & -11.37 & -12.22 \\
$\zeta=5$ & & & & 7.59 & 7.53 & 6.55 & -2.62 & -2.11 & -2.63 \\
$\zeta=6$ & & & & & & & 1.75 & 1.46 & 0.49 \\
\hline
\end{tabular}

\begin{tabular}{|c|c|c|c|c|c|c|c|c|c|c|c|c|c|c|c|}
\hline \multirow[b]{2}{*}{ The $p f$-shell } & \multicolumn{3}{|c|}{$k=1$} & \multicolumn{3}{|c|}{$k=2$} & \multicolumn{3}{|c|}{$k=3$} & \multicolumn{3}{|c|}{$k=4$} & \multicolumn{3}{|c|}{$k=5$} \\
\hline & SP & SSP & SPM & SP & SSP & SPM & SP & SSP & SPM & SP & SSP & SPM & SP & SSP & SPM \\
\hline$\zeta=1$ & -23.10 & -23.10 & -23.10 & -43.31 & -43.31 & -43.31 & -60.67 & -60.67 & -60.67 & -75.13 & -75.13 & -75.13 & -87.59 & -87.59 & -87.59 \\
\hline$\zeta=2$ & -11.45 & -12.46 & -12.75 & -34.45 & -34.36 & -34.43 & -54.58 & -53.57 & -53.50 & -71.86 & -70.31 & -70.19 & -83.99 & -82.17 & -82.32 \\
\hline$\zeta=3$ & -8.29 & -8.67 & -8.82 & -31.36 & -30.84 & -30.71 & -51.57 & -50.26 & -49.96 & -68.92 & -67.01 & -66.64 & -83.54 & -81.82 & -81.56 \\
\hline$\zeta=4$ & -2.66 & -3.50 & -4.13 & -25.95 & -26.17 & -26.43 & -46.17 & -45.81 & -45.83 & -65.79 & -63.54 & -63.50 & -80.61 & -77.94 & -77.76 \\
\hline$\zeta=5$ & & & & -22.80 & -23.98 & -24.40 & -45.72 & -44.97 & -45.08 & -63.54 & -62.62 & -63.56 & -78.38 & -75.86 & -75.58 \\
\hline$\zeta=6$ & & & & -19.73 & -21.01 & -21.36 & -42.72 & -42.15 & -42.12 & -62.85 & -60.76 & -60.53 & -77.47 & -75.18 & -75.49 \\
\hline$\zeta=7$ & & & & -14.33 & -16.18 & -17.02 & -37.32 & -37.53 & -37.93 & -57.45 & -56.22 & -58.39 & -77.11 & -73.17 & -72.94 \\
\hline$\zeta=8$ & & & & -11.18 & -12.52 & -13.42 & -34.22 & -34.09 & -34.50 & -54.43 & -52.94 & -53.15 & -75.02 & -72.13 & -71.89 \\
\hline$\zeta=9$ & & & & -5.69 & -6.82 & -7.68 & -31.09 & -32.67 & -33.29 & -54.00 & -52.91 & -53.04 & -74.52 & -71.91 & -71.75 \\
\hline$\zeta=10$ & & & & & & & -28.73 & -28.61 & -28.91 & -48.93 & -48.12 & -48.60 & -72.05 & -68.22 & -67.86 \\
\hline$\zeta=11$ & & & & & & & -25.69 & -27.73 & -28.68 & -48.59 & -47.55 & -47.54 & -71.40 & -67.92 & -67.80 \\
\hline$\zeta=12$ & & & & & & & -22.62 & -24.86 & -25.98 & -45.59 & -45.39 & -45.98 & -68.95 & -66.47 & -66.68 \\
\hline$\zeta=13$ & & & & & & & -17.14 & -19.18 & -20.22 & -40.09 & -39.86 & -40.33 & -65.97 & -65.97 & -66.30 \\
\hline$\zeta=14$ & & & & & & & -13.98 & -15.57 & -16.69 & -37.00 & -36.43 & -37.74 & -65.46 & -63.02 & -62.81 \\
\hline$\zeta=15$ & & & & & & & -8.42 & -9.25 & -9.91 & -33.98 & -36.43 & -36.93 & -62.77 & -61.36 & -61.57 \\
\hline$\zeta=16$ & & & & & & & & & & -31.42 & -30.63 & -31.72 & -62.57 & -59.07 & -59.25 \\
\hline$\zeta=17$ & & & & & & & & & & -28.50 & -30.29 & -30.30 & -59.97 & -58.84 & -58.92 \\
\hline$\zeta=18$ & & & & & & & & & & -25.43 & -27.76 & -28.98 & -57.16 & -56.40 & -56.52 \\
\hline$\zeta=19$ & & & & & & & & & & -19.86 & -21.45 & -22.18 & -54.02 & -54.88 & $8-55.89$ \\
\hline$\zeta=20$ & & & & & & & & & & -16.71 & -17.87 & -18.64 & -50.98 & -52.70 & $0-53.03$ \\
\hline$\zeta=21$ & & & & & & & & & & & & & -45.61 & -49.32 & $2-49.91$ \\
\hline$\zeta=22$ & & & & & & & & & & & & & -42.53 & -45.57 & $7-46.37$ \\
\hline
\end{tabular}

\section{SUMMARY}

The exact solution of nuclear spherical mean-field plus a special orbit-dependent separable pairing model is revisited by using the algebraic Bethe ansatz method, of which the separable pairing interaction parameters is obtained by a linear fitting in terms of the single-particle energies. The special separable pairing model studied in this work belong to a special family of the hyperbolic Richardson-Gaudin models proposed previously [31, 32]. The advantage of the model is that, similar to the standard pairing case, it can be solved easily by using the extended Heine-Stieltjes polynomial approach. We have shown that this special separable pairing model indeed provides similar pair structures of the model with the general separable pairing interaction [27]. Though there are deviations from the general separable pairing model, the special separable pairing model is obviously better than the standard pairing model in many aspects. As demonstrated in [34], the model is indeed suitable to describe many-particle correlations in pair-rich heavy nuclei.

Support from the U.S. National Science Foundation (OCI-0904874, ACI-1516338), U.S. Department of Energy (DE- 
SC0005248), the Southeastern Universities Research Association, the China-U.S. Theory Institute for Physics with Exotic Nuclei (CUSTIPEN) (DE-SC0009971), the National Natural Science Foundation of China (11375080), and the LSU-LNNU joint research program (9961) is acknowledged.

[1] J. Bardeen, L. N. Cooper, J. R. Schrieffer, Phys. Rev. 108, 1175 (1957).

[2] M. Randeria, J. M. Duan, L. Y. Shieh, Phys. Rev. Lett. 62, 981 (1989) 981.

[3] D. W. Cooper, J. S. Batchelder, M. A. Taubenblatt, J. Coll. Int. Sci. 144, 201 (1991).

[4] K. K. Gomes, A. N. Pasupathy, A. Pushp, S. Ono, Y. Ando, and A. Yazdani, Nature 447, 569-572 (2007).

[5] P. Ring and P. Schuck, The Nuclear Many-Body Problem (Springer Verleg, Berlin, 1980).

[6] A. Bohr, B. R. Mottelson, and D. Pines, Phys. Rev. 110, 936 (1958); S. T. Belyaev, Mat. Fys. Medd. K. Dan. Vidensk. Selsk. 31, 11 (1959).

[7] M. Hasegawa and S. Tazaki, Phys. Rev. C 47, 188 (1993).

[8] H. C. Pradhan, Y. Nogami, and J. Law, Nucl. Phys. A 201, 357(1973).

[9] H. J. Mang, Phys. Rep. 18, 325 (1975).

[10] G. D. Dans and A. Klein, Phys. Rev. 143, 735 (1966).

[11] A. Covello and E. Salusti, Phys. Rev. 162, 859 (1967).

[12] M. Bishari, I. Unna, and A. Mann, Phys. Rev. C 3, 1715 (1971).

[13] J. Y. Zeng, C. S. Cheng, Nucl. Phys. A 405, 1 (1983); 411, 49 (1984); 414, 253 (1984).

[14] H. Molique and J. Dudek, Phys. Rev. C 56, 1795 (1997).

[15] A. Volya, B. A. Brown, and V. Zelevinsky, Phys. Lett. B 509, 37 (2001).

[16] A. K. Kerman and R. D. Lawson, Phys. Rev. 124, 162 (1961).

[17] V. Zelevinsky and A. Volya, Physics of Atomic Nuclei 66, 1781 (2003).

[18] M. Gaudin, J. Physique 37, 1087 (1976).

[19] R. W. Richardson, Phys. Lett. 3, 277 (1963); 5, 82 (1963); R. W. Richardson and N. Sherman, Nucl. Phys. 52, 221 (1964); 52, 253 (1964).

[20] J. Dukelsky, S. Pittel, and G. Sierra, Rev. Mod. Phys. 76, 643 (2004).

[21] F. Pan, L. Bao, L. Zhai, X. Cui, and J. P. Draayer, J. Phys. A: Math. Theor. 44, 395305 (2011).

[22] X. Guan, K. D. Launey, M. Xie, L. Bao, F. Pan, J. P. Draayer, Phys. Rev. C 86, 024313 (2012).

[23] X. Guan, K. D. Launey, M. Xie, L. Bao, F. Pan, J. P. Draayer, Comp. Phys. Commun. 185, 2714 (2014).

[24] C. Qi and T. Chen, Phys. Rev. C 92, 051304(R) (2015).

[25] F. Pan, V. G. Gueorguiev, and J. P. Draayer, Phys. Rev. Lett. 92, 112503 (2004).

[26] F. Pan, X. Ding, K. D. Launey, H. Li, X. Xu, J. P. Draayer, Nucl. Phys. A 947, 234 (2016).

[27] F. Pan, J. P. Draayer, and W. E. Ormand, Phys. Lett. B 422, 1 (1998).

[28] A. B. Balantekin and Y. Pehlivan, Phys. Rev. C 76, 051001(R) (2007).

[29] F. Pan and J. P. Draayer, Phys. Lett. B 442, 7 (1998).

[30] F. Pan and J. P. Draayer, Ann. Phys. (N. Y. ) 271, 120 (1999).

[31] S. M. A. Rombouts, J. Dukelsky, and G. Ortiz, Phys. Rev. B 82224510 (2010).

[32] P. W. Claeys, S. De Baerdemacker, M. Van Raemdonck, and D. Van Neck, Phys. Rev. B 91, 155102 (2015).

[33] F. Pan, M. Xie, X. Guan, L. Dai, and J. P. Draayer, Phys. Rev. C 80, 044306 (2009).

[34] J. Dukelsky, S. Lerma H., L. M. Robledo, R. Rodriguez-Guzman, and S. M. A. Rombouts, Phys. Rev. C 84, 061301 (2011).

[35] F. Nowacki and A. Poves, Phys. Rev. C 79, 014310 (2009).

[36] M. Honma, T. Otsuka, B. A. Brown, and T. Mizusaki, Phys. Rev. C 69, 034335 (2004). 